\title{
FLUID FLOW IN AN ASYMMETRIC CHANNEL
}

\author{
P. MUTHU AND TESFAHUN BERHANE
}

\begin{abstract}
In this paper, we investigate steady, viscous, incompressible fluid flow in an asymmetric channel with absorbing walls which may have possible applications in flows in renal tubules. The effect of fluid absorption through permeable wall is accounted for by prescribing flux as a function of axial distance. The nonlinear governing equations of motion are linearized by perturbation method by assuming $\delta$ (ratio of inlet width to wavelength) as a small parameter and the resulting equations are solved by numerical methods. The effects of reabsorption coefficient $(\alpha)$ and phase difference $(\phi)$ on the velocity profiles, mean pressure drop and wall shear stress are studied and explained graphically.
\end{abstract}

\section{Introduction}

The kidneys play key roles in body function, not only by filtering the blood and getting rid of waste products, but also by balancing levels of electrolytes in the body, controlling blood pressure, and stimulating the production of red blood cells. The basic functional unit of kidney is nephron. Each kidney contains over a million tiny units (of nephrons), all similar in structure and function. Each nephron functions independently and in most instances it is sufficient to study the function of nephron to understand the mechanism of kidney in terms of mathematical models. In nephrons, the portion after the Bowman's capsule is called proximal convoluted tubule. It is the place where most of the wanted substances, like water, glucose and electrolytes are reabsorbed back into the plasma and unwanted substances pass into urine. Thus it is of interest to study the flow in proximal tubule using mathematical models.

Various authors considered flow in renal tubules. Macey [1] was the first to study the mathematical modeling of the flow in proximal renal tubule. He formulated the problem as the flow of an incompressible viscous fluid through a circular tube with linear rate of reabsorption at the wall. Kelman [2] noted that the bulk flow in the proximal tubule decays exponentially with the axial distance. Later, Macey [3] used this condition and solved the equations of motion to find average pressure drop. Marshall and Trowbridge [4] and Palatt et.al [5]

Corresponding author: P. Muthu.

2000 Mathematics Subject Classification. .

Key words and phrases. Asymmetric channel, perturbation method, permeable wall. 
used physical conditions existing at the permeable wall instead of prescribing the flux/radial velocity at the wall.

In all the above studies the renal tubule is assumed as cylindrical tube of uniform crosssection, while in general such tubes may not have uniform cross-section throughout their length. Radhakrishnamacharya et al [6] made an attempt to understand the flow through the renal tubule by studying the hydrodynamical aspects of an incompressible viscous fluid in a circular tube of varying cross-section with reabsorption at the wall. Chandra and Prasad [7] analyzed flow in rigid tubes of slowly varying cross-section with absorbing wall. Chaturani and Ranganatha [8] considered fluid flow through a diverging/converging tube with variable wall permeability. Recently, Muthu and Tesfahun [9] considered the effects of slope parameter and reabsorption coefficient on the flow of fluid in a symmetric channel with varying cross section.

All the above literature considered the geometry of the renal tubule to be of symmetric. But in nature renal tubules may not be symmetric in all their length so we need to consider the asymmetric channel. In this paper, we have made an attempt to understand the flow through renal tubule by studying the hydrodynamical aspect of an incompressible viscous fluid in an asymmetric channel of varying cross-section with reabsorption at the walls. The boundary of the channel walls are taken as

$$
\begin{array}{lll}
\eta_{1}(x)=d_{1}+a_{1} \cos \left(\frac{2 \pi x}{\lambda}\right) & \ldots & \text { upper wall } \\
\eta_{2}(x)=-d_{2}-b_{1} \cos \left(\frac{2 \pi x}{\lambda}+\phi\right) & \ldots & \text { lower wall }
\end{array}
$$

where $d_{1}$ and $d_{2}$ are the half width of the channel from the x-axis to $\eta_{1}(x)$ and $\eta_{2}(x)$ respectively at the inlet (at $x=0$ ), $a_{1}$ and $b_{1}$ are amplitudes and $\lambda$ is the wave length, and further $a_{1}, b_{1}, d_{1}, d_{2}$ and $\phi$ satisfies the condition [10] - [11]

$$
a_{1}^{2}+b_{1}^{2}+2 a_{1} b_{1} \cos (\phi) \leq\left(d_{1}+d_{2}\right)^{2}
$$

\section{Mathematical Formulation}

Consider an incompressible fluid flow through asymmetric channel with slowly varying cross-section as given by equation (1). The motion of the fluid is assumed to be laminar and steady. The channel is long enough to neglect the initial and end effects. The governing equations of such fluid motion are given by

$$
\frac{\partial u}{\partial x}+\frac{\partial v}{\partial y}=0
$$




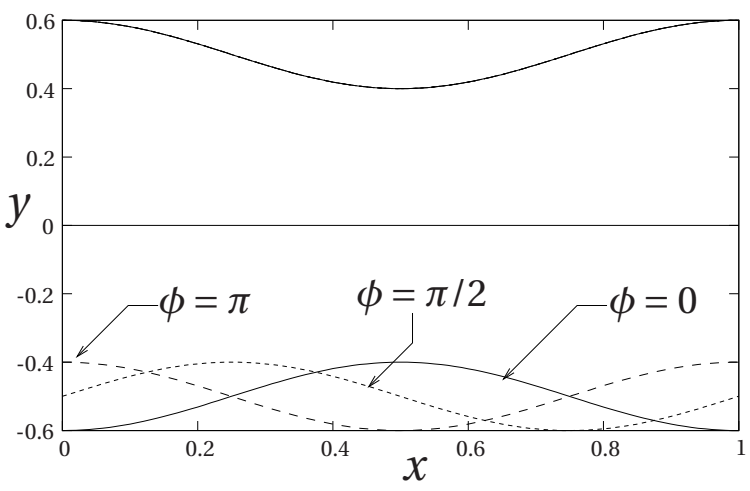

Figure 1: Two dimensional non-symmetric geometry of renal tubules.

$$
\begin{aligned}
& u \frac{\partial u}{\partial x}+v \frac{\partial u}{\partial y}=-\frac{1}{\rho} \frac{\partial p}{\partial x}+v\left(\frac{\partial^{2} u}{\partial x^{2}}+\frac{\partial^{2} u}{\partial y^{2}}\right) \\
& u \frac{\partial v}{\partial x}+v \frac{\partial v}{\partial y}=-\frac{1}{\rho} \frac{\partial p}{\partial y}+v\left(\frac{\partial^{2} v}{\partial x^{2}}+\frac{\partial^{2} v}{\partial y^{2}}\right)
\end{aligned}
$$

where $u$ and $v$ are the velocity components along the $x$ and $y$ axes respectively, $p$ is the pressure, $\rho$ density of the fluid and $v=\frac{\mu}{\rho}$ is kinematic viscosity.

The boundary conditions are taken as follows:

(a) The tangential velocity at the wall is zero. That is,

$$
\begin{array}{llll}
u+\frac{d \eta_{1}}{d x} v=0 & \text { at } & y & =\eta_{1}(x) . \\
u+\frac{d \eta_{2}}{d x} v=0 & \text { at } & y & =\eta_{2}(x) .
\end{array}
$$

(b) The reabsorption has been accounted for by considering the bulk flow as a decreasing function of $x$. That is, the flux across a cross-section is given as

$$
Q(x)=\int_{\eta_{2}(x)}^{\eta_{1}(x)} u(x, y) d y=Q_{0} F(\alpha x),
$$

where $F(\alpha x)=1$ when $\alpha=0$ and decreases with $x, \alpha \geq 0$ is the reabsorption coefficient and is a constant, and $Q_{0}$ is the flux across the cross-section at $x=0$.

Introducing stream function $\psi$ by

$$
u=\frac{\partial \psi}{\partial y} \quad \text { and } \quad v=-\frac{\partial \psi}{\partial x}
$$

and the following non-dimensional quantities

$$
x^{\prime}=\frac{x}{\lambda}, \quad y^{\prime}=\frac{y}{d}, \quad \eta_{1}^{\prime}=\frac{\eta_{1}}{d},
$$




$$
\eta_{2}^{\prime}=\frac{\eta_{2}}{d}, \quad \psi^{\prime}=\frac{\psi}{Q_{0}}, \quad \alpha^{\prime}=\alpha \lambda, \quad p^{\prime}=\frac{d^{2}}{\mu Q_{0}} p
$$

where $d=d_{1}+d_{2}$, equations (3) - (5) are transformed to the non-dimensional form as (after dropping the primes) :

$$
\left(\delta^{2} \frac{\partial^{2}}{\partial x^{2}}+\frac{\partial^{2}}{\partial y^{2}}\right)^{2} \psi=\delta R\left[\frac{\partial \psi}{\partial y}\left(\delta^{2} \frac{\partial^{2}}{\partial x^{2}}+\frac{\partial^{2}}{\partial y^{2}}\right) \frac{\partial \psi}{\partial x}-\frac{\partial \psi}{\partial x}\left(\delta^{2} \frac{\partial^{2}}{\partial x^{2}}+\frac{\partial^{2}}{\partial y^{2}}\right) \frac{\partial \psi}{\partial y}\right]
$$

where $\delta=\frac{d}{\lambda}$ and $R=\frac{Q_{0}}{v}$.

Further the boundary conditions (6) - (8) become

$$
\begin{array}{rlc}
\frac{\partial \psi}{\partial y}=\delta A \sin (2 \pi x) \frac{\partial \psi}{\partial x} \quad \text { at } & y=\eta_{1}(x)=\beta_{1}+\epsilon_{1} \cos (2 \pi x), \\
\frac{\partial \psi}{\partial y}=\delta B \sin (2 \pi x+\phi) \frac{\partial \psi}{\partial x} & \text { at } & y=\eta_{2}(x)=\beta_{2}+\epsilon_{2} \cos (2 \pi x+\phi), \\
\psi=F(\alpha x) & \text { at } & y=\eta_{1}(x)=\beta_{1}+\epsilon_{1} \cos (2 \pi x) \\
\text { and } \psi=-F(\alpha x) & \text { at } & y=\eta_{2}(x)=\beta_{2}+\epsilon_{2} \cos (2 \pi x+\phi)
\end{array}
$$

where $A=-\frac{2 \pi a_{1}}{\lambda}, B=\frac{2 \pi b_{1}}{\lambda}, \epsilon_{1}=\frac{a_{1}}{d}, \epsilon_{2}=-\frac{b_{1}}{d}, \beta_{1}=\frac{d_{1}}{d}$ and $\beta_{2}=-\frac{d_{2}}{d}$.

The parameter $R$ is the Reynolds number and $\delta$ is the wave-number (the ratio of inlet width to the wavelength). $\epsilon_{1}$ and $\epsilon_{2}$ are amplitude ratios (the ratios of amplitudes $a_{1}$ and $b_{1}$ to the inlet width respectively) and $\beta_{1}$ and $\beta_{2}$ are ratios of distance from $\mathrm{x}$-axis to upper wall and lower wall to the inlet width respectively. In this problem, we consider exponentially decaying bulk flow [6], that is, in equation (8), $F$ is taken as

$$
F(\alpha x)=e^{-\alpha x}
$$

\section{Method of Solution}

It may be noted that the flow is quite complex because of nonlinearity of governing equation and the boundary conditions. To solve equation (10) for velocity components, in the present analysis, we assume the wave number $\delta \ll 1$ (long wavelength approximation). We shall seek a solution for stream function $\psi(x, y)$ in the form of a power series in terms of $\delta$, as

$$
\psi(x, y)=\psi_{0}(x, y)+\delta \psi_{1}(x, y)+\ldots . .
$$

Substituting equation (16) in equations (10) - (14) and collecting coefficients of various like powers of $\delta$, we get the following sets of equations for $\psi_{0}(x, y), \psi_{1}(x, y), \cdots$.

$\delta^{0}$ Case :

$$
\frac{\partial^{4} \psi_{0}}{\partial y^{4}}=0
$$


The boundary conditions are :

$$
\begin{aligned}
& \frac{\partial \psi_{0}}{\partial y}=0 \quad \text { at } \quad y=\eta_{1}(x) \quad \text { and } \quad y=\eta_{2}(x) \\
& \psi_{0}=F(\alpha x)=e^{-\alpha x} \quad \text { at } \quad y=\eta_{1}(x) \\
& \psi_{0}=-F(\alpha x)=-e^{-\alpha x} \quad \text { at } \quad y=\eta_{2}(x)
\end{aligned}
$$

$\delta^{1}$ Case :

$$
\frac{\partial^{4} \psi_{1}}{\partial y^{4}}=R\left[\frac{\partial \psi_{0}}{\partial y} \frac{\partial^{3} \psi_{0}}{\partial y^{2} \partial x}-\frac{\partial \psi_{0}}{\partial x} \frac{\partial^{3} \psi_{0}}{\partial y^{3}}\right]
$$

The boundary conditions are :

$$
\begin{array}{ccc}
\frac{\partial \psi_{1}}{\partial y}=A \sin (2 \pi x) \frac{\partial \psi_{0}}{\partial x} \quad \text { at } & y=\eta_{1}(x) \\
\frac{\partial \psi_{1}}{\partial y}=B \sin (2 \pi x+\phi) \frac{\partial \psi_{0}}{\partial x} & \text { at } & y=\eta_{2}(x) \\
\psi_{1}=0 \quad \text { at } \quad y=\eta_{1}(x) \text { and } & y=\eta_{2}(x)
\end{array}
$$

Similar expressions can be written for higher orders of $\delta$. However, since we are looking for an approximate analytical solution for the problem, we consider up to order of $\delta^{1}$ equations.

The solution of equation (17) along with equations (18) to (20) is

$$
\psi_{0}(x, y)=A_{1} y^{3}+A_{2} y^{2}+A_{3} y+A_{4}
$$

where

$$
\begin{aligned}
& A_{1}(x)=\frac{4 e^{-\alpha x}}{\left(\eta_{2}-\eta_{1}\right)^{3}}, \\
& A_{2}(x)=\frac{-6\left(\eta_{2}+\eta_{1}\right) e^{-\alpha x}}{\left(\eta_{2}-\eta_{1}\right)^{3}}, \\
& A_{3}(x)=\frac{12\left(\eta_{2} \eta_{1}\right) e^{-\alpha x}}{\left(\eta_{2}-\eta_{1}\right)^{3}}, \\
& A_{4}(x)=\frac{\left(\eta_{2}^{2}-3 \eta_{2}^{2} \eta_{1}-3 \eta_{2} \eta_{1}^{2}+\eta_{1}^{3}\right) e^{-\alpha x}}{\left(\eta_{2}-\eta_{1}\right)^{3}} .
\end{aligned}
$$

The solution of equation (21) along with equations (22) to (24) is

$$
\psi_{1}(x, y)=\frac{A_{5}}{1680} y^{8}+\frac{A_{6}}{840} y^{7}+\frac{A_{7}}{360} y^{6}+\frac{A_{8}}{120} y^{5}+\frac{A_{9}}{24} y^{4}+A_{10}+A_{11} y+A_{12} y^{2}+A_{13} y^{3}
$$


where

$$
\begin{aligned}
& A_{5}(x)=18 R A_{1} \frac{d A_{1}}{d x} \\
& A_{6}(x)=R\left(12 A_{2} \frac{d A_{1}}{d x}-6 A_{1} \frac{d A_{1}}{d x}\right), \\
& A_{7}(x)=6 A_{3} \frac{d A_{1}}{d x} \\
& A_{8}(x)=4 A_{2} \frac{d A_{2}}{d x}-6 A_{1} \frac{d A_{3}}{d x}, \\
& A_{9}(x)=2 A_{3} \frac{d A_{2}}{d x}-6 A_{1} \frac{d A_{4}}{d x}, \\
& A_{10}(x)=\frac{1}{5040\left(\eta_{2}-\eta_{1}\right)^{2}}\left\{\eta _ { 2 } \eta _ { 1 } \left\{3 \eta _ { 2 } \eta _ { 1 } ( \eta _ { 2 } - \eta _ { 1 } ) ^ { 2 } \left(5 \eta_{2}{ }^{4}+8 \eta_{2}{ }^{3} \eta_{1}+9 \eta_{2}{ }^{2} \eta_{1}{ }^{2}+8 \eta_{2} \eta_{1}{ }^{3}\right.\right.\right. \\
& \left.+5 \eta_{1}^{4}\right) A_{5}-2\left[-6 \eta_{2} \eta_{1}\left(\eta_{2}-\eta_{1}\right)^{2}\left(2 \eta_{2}{ }^{3}+3 \eta_{2}{ }^{2} \eta_{1}+3 \eta_{2} \eta_{1}{ }^{2}+2 \eta_{1}{ }^{3}\right) A_{6}\right. \\
& +7\left[-\eta_{2} \eta_{1}\left(\eta_{2}-\eta_{1}\right)^{2}\left(3 \eta_{2}^{2}+4 \eta_{2} \eta_{1}+3 \eta_{1}^{2}\right) A_{7}+3\left(-2 \eta_{2} \eta_{1}\left(\eta_{2}-\eta_{1}\right)^{2}\left(\eta_{2}+\eta_{1}\right) A_{8}\right.\right. \\
& +5\left(2 4 \left(A \eta_{2} \sin (2 \pi x)\left[\frac{d A_{4}}{d x}+\eta_{1} \frac{d A_{3}}{d x}+\eta_{1}^{2}\left(\frac{d A_{2}}{d x}+\frac{d A_{1}}{d x} \eta_{1}\right)\right]\right.\right. \\
& \left.+B \eta_{1} \sin (2 \pi x+\phi)\left[\frac{d A_{4}}{d x}+\eta_{2} \frac{d A_{3}}{d x}+\eta_{2}^{2}\left(\frac{d A_{2}}{d x}+\frac{d A_{1}}{d x} \eta_{2}\right)\right]\right) \\
& \left.\left.\left.\left.\left.\left.-\eta_{2} \eta_{1}\left(\eta_{2}-\eta_{1}\right)^{2} A_{9}\right)\right)\right]\right]\right\}\right\} \\
& A_{11}(x)=\frac{-1}{840\left(\eta_{2}-\eta_{1}\right)^{2}}\left\{\eta _ { 2 } \eta _ { 1 } ( \eta _ { 2 } - \eta _ { 1 } ) ^ { 2 } \left(5 \eta_{2}{ }^{5}+11 \eta_{2}{ }^{4} \eta_{1}+14 \eta_{2}{ }^{3} \eta_{1}{ }^{2}+14 \eta_{2}{ }^{2} \eta_{1}{ }^{3}+11 \eta_{2} \eta_{1}{ }^{4}\right.\right. \\
& \left.+5 \eta_{1}{ }^{5}\right) A_{5}+\eta_{2} \eta_{1}\left(\eta_{2}-\eta_{1}\right)^{2}\left(8 \eta_{2}{ }^{4}+17 \eta_{2}{ }^{3} \eta_{1}+20 \eta_{2}{ }^{2} \eta_{1}{ }^{2}+17 \eta_{2} \eta_{1}{ }^{3}+8 \eta_{1}^{4}\right) A_{6} \\
& -7\left[-2 \eta_{2} \eta_{1}\left(3 \eta_{2}^{5}-\eta_{2}{ }^{3} \eta_{1}{ }^{2}-\eta_{2}{ }^{2} \eta_{1}{ }^{3}+\eta_{1}{ }^{5}\right) A_{7}-\eta_{2} \eta_{1}\left(\eta_{2}-\eta_{1}\right)^{2}\left(4 \eta_{2}{ }^{2}+7 \eta_{2} \eta_{1}\right.\right. \\
& \left.+4 \eta_{1}^{2}\right) A_{8}+10\left(1 2 \left(A \eta_{2}\left(\eta_{2}+2 \eta_{1}\right) \sin (2 \pi x)\left[\frac{d A_{4}}{d x}+\eta_{1} \frac{d A_{3}}{d x}+\eta_{1}^{2}\left(\frac{d A_{2}}{d x}+\frac{d A_{1}}{d x} \eta_{1}\right)\right]\right.\right. \\
& \left.+B \eta_{1}\left(2 \eta_{2}+\eta_{1}\right) \sin (2 \pi x+\phi)\left[\frac{d A_{4}}{d x}+\eta_{2} \frac{d A_{3}}{d x}+\eta_{2}^{2}\left(\frac{d A_{2}}{d x}+\frac{d A_{1}}{d x} \eta_{2}\right)\right]\right) \\
& \left.\left.\left.-\eta_{2} \eta_{1}\left(\eta_{2}+\eta_{1}\right) A_{9}\right)\right]\right\} \\
& A_{12}(x)=\frac{-1}{1680\left(\eta_{2}-\eta_{1}\right)^{2}}\left\{( \eta _ { 2 } - \eta _ { 1 } ) ^ { 2 } \left(5 \eta_{2}{ }^{6}+20 \eta_{2}{ }^{5} \eta_{1}+29 \eta_{2}{ }^{4} \eta_{1}{ }^{2}+32 \eta_{2}{ }^{3} \eta_{1}{ }^{3}+29 \eta_{2}{ }^{2} \eta_{1}{ }^{4}\right.\right. \\
& \left.+20 \eta_{2} \eta_{1}{ }^{5}+5 \eta_{1}{ }^{6}\right) A_{5}-\left[-2\left(\eta_{2}-\eta_{1}\right)^{2}\left(2 \eta_{2}{ }^{5}+8 \eta_{2}{ }^{4} \eta_{1}+11 \eta_{2}{ }^{3} \eta_{1}{ }^{2}+11 \eta_{2}{ }^{2} \eta_{1}{ }^{3}\right.\right. \\
& \left.+8 \eta_{2} \eta_{1}{ }^{4}+2 \eta_{1}{ }^{5}\right) A_{6}+7\left(-\left(\eta_{2}-\eta_{1}\right)^{2}\left(\eta_{2}{ }^{4}+4 \eta_{2}{ }^{3} \eta_{1}+5 \eta_{2}{ }^{2} \eta_{1}{ }^{2}+4 \eta_{2} \eta_{1}{ }^{3}+\eta_{1}{ }^{4}\right) A_{7}\right. \\
& -2\left(\eta_{2}-\eta_{1}\right)^{2}\left(\eta_{2}{ }^{3}+4 \eta_{2}{ }^{2} \eta_{1}+4 \eta_{2} \eta_{1}{ }^{2}+\eta_{1}{ }^{3}\right) A_{8}+5\left(2 4 \left(A ( 2 \eta _ { 2 } + \eta _ { 1 } ) \operatorname { s i n } ( 2 \pi x ) \left[\frac{d A_{4}}{d x}\right.\right.\right.
\end{aligned}
$$




$$
\begin{aligned}
& \left.+\eta_{1} \frac{d A_{3}}{d x}+\eta_{1}{ }^{2}\left(\frac{d A_{2}}{d x}+\frac{d A_{1}}{d x} \eta_{1}\right)\right]+B\left(\eta_{2}+2 \eta_{1}\right) \sin (2 \pi x+\phi)\left[\frac{d A_{4}}{d x}+\eta_{2} \frac{d A_{3}}{d x}\right. \\
& \left.\left.\left.\left.\left.\left.+\eta_{2}{ }^{2}\left(\frac{d A_{2}}{d x}+\frac{d A_{1}}{d x} \eta_{2}\right)\right]\right)-\left(\eta_{2}-\eta_{1}\right)^{2}\left(\eta_{2}{ }^{2}+4 \eta_{2} \eta_{1}+\eta_{1}{ }^{2}\right) A_{9}\right)\right)\right]\right\} \\
A_{13}(x)= & \frac{-1}{2520\left(\eta_{2}-\eta_{1}\right)^{2}}\left\{-3\left(\eta_{2}-\eta_{1}\right)^{2}\left(3 \eta_{2}{ }^{5}+5 \eta_{2}{ }^{4} \eta_{1}+6 \eta_{2}{ }^{3} \eta_{1}{ }^{2}+6 \eta_{2}{ }^{2} \eta_{1}{ }^{3}+5 \eta_{2} \eta_{1}{ }^{4}\right.\right. \\
& \left.+3 \eta_{1}^{5}\right) A_{5}-3\left(\eta_{2}-\eta_{1}\right)^{2}\left(5 \eta_{2}{ }^{4}+8 \eta_{2}{ }^{3} \eta_{1}+9 \eta_{2}{ }^{2} \eta_{1}{ }^{2}+8 \eta_{2} \eta_{1}{ }^{3}+5 \eta_{1}{ }^{4}\right) A_{6} \\
& +7\left[-2\left(\eta_{2}-\eta_{1}\right)^{2}\left(2 \eta_{2}{ }^{3}+3 \eta_{2}{ }^{2} \eta_{1}+3 \eta_{2} \eta_{1}{ }^{2}+2 \eta_{1}{ }^{3}\right) A_{7}\right. \\
& -3\left(\eta_{2}-\eta_{1}\right)^{2}\left(\eta_{2}{ }^{2}+4 \eta_{2} \eta_{1}+3 \eta_{1}{ }^{2}\right) A_{8} \\
& +30\left(1 2 \left(A \sin (2 \pi x)\left[\frac{d A_{4}}{d x}+\eta_{1} \frac{d A_{3}}{d x}+\eta_{1}{ }^{2}\left(\frac{d A_{2}}{d x}+\frac{d A_{1}}{d x} \eta_{1}\right)\right]\right.\right. \\
& \left.\left.\left.\left.+B \sin (2 \pi x+\phi)\left[\frac{d A_{4}}{d x}+\eta_{2} \frac{d A_{3}}{d x}+\eta_{2}{ }^{2}\left(\frac{d A_{2}}{d x}+\frac{d A_{1}}{d x} \eta_{2}\right)\right]\right)-\left(\eta_{2}-\eta_{1}\right)^{2}\left(\eta_{2}+\eta_{1}\right) A_{9}\right)\right]\right\}
\end{aligned}
$$

Hence, substituting $\psi_{0}$ and $\psi_{1}$ in equation (16), we get that

$$
\begin{aligned}
\psi(x, y)= & A_{1} y^{3}+A_{2} y^{2}+A_{3} y+A_{4} \\
& +\delta\left(\frac{A_{5}}{1680} y^{8}+\frac{A_{6}}{840} y^{7}+\frac{A_{7}}{360} y^{6}+\frac{A_{8}}{120} y^{5}+\frac{A_{9}}{24} y^{4}+A_{10}+A_{11} y+A_{12} y^{2}+A_{13} y^{3}\right) .
\end{aligned}
$$

Now, the nondimensional pressure $p(x, y)$ can be obtained by using equations (27), (9) and (4). It is given as

$$
p(x, y)=\delta \frac{\partial u}{\partial x}+\frac{1}{\delta} \int \frac{\partial^{2} u}{\partial y^{2}} d x-R\left(\int u \frac{\partial u}{\partial x} d x+\int v \frac{\partial u}{\partial y} d x\right) .
$$

The mean pressure is given as

$$
\bar{p}(x)=\frac{1}{\eta_{1}(x)-\eta_{2}(x)} \int_{\eta_{2}(x)}^{\eta_{1}(x)} p(x, y) d y
$$

Further, the mean pressure drop between $x=0$ and $x=x_{0}$ is

$$
\Delta \bar{p}\left(x_{0}\right)=\bar{p}(0)-\bar{p}\left(x_{0}\right) .
$$

The wall shear stress $\tau_{w}(x)$ is defined as

$$
\tau_{w}(x)=\frac{\left(\sigma_{y y}-\sigma_{x x}\right) \frac{d y}{d x}+\sigma_{x y}\left[1-\left(\frac{d y}{d x}\right)^{2}\right]}{1+\left(\frac{d y}{d x}\right)^{2}} \quad \text { at } y=\eta_{1}(x) \text { and } y=\eta_{2}(x)
$$

where $\sigma_{x x}=2 \mu \frac{\partial u}{\partial x}, \sigma_{y y}=2 \mu \frac{\partial v}{\partial y}$, and $\quad \sigma_{x y}=\mu\left(\frac{\partial u}{\partial y}+\frac{\partial v}{\partial x}\right)$. 
Using the nondimensional quantity $\tau_{w 1}^{\prime}=\frac{d^{2}}{\mu Q_{0}} \tau_{w 1}$ and $\tau_{w 2}^{\prime}=\frac{d^{2}}{\mu Q_{0}} \tau_{w 2}$, the wall shear stress $\tau_{w 1}$ and $\tau_{w 2}$ become (after dropping the prime),

$$
\begin{gathered}
\tau_{w 1}=\frac{2 \delta^{2}\left(\frac{\partial v}{\partial y}-\frac{\partial u}{\partial x}\right) \frac{d \eta_{1}}{d x}+\left(\frac{\partial u}{\partial y}+\delta^{2} \frac{\partial v}{\partial x}\right)\left[1-\delta^{2}\left(\frac{d \eta_{1}}{d x}\right)^{2}\right]}{1+\delta^{2}\left(\frac{d \eta_{1}}{d x}\right)^{2}} \\
\tau_{w 2}=\frac{2 \delta^{2}\left(\frac{\partial v}{\partial y}-\frac{\partial u}{\partial x}\right) \frac{d \eta_{2}}{d x}+\left(\frac{\partial u}{\partial y}+\delta^{2} \frac{\partial v}{\partial x}\right)\left[1-\delta^{2}\left(\frac{d \eta_{2}}{d x}\right)^{2}\right]}{1+\delta^{2}\left(\frac{d \eta_{2}}{d x}\right)^{2}} .
\end{gathered}
$$

It may be noted that in equation (28), the integrals are difficult to evaluate analytically to get closed form expression for $p(x, y)$. Therefore, they are calculated by numerical integration.

\section{Results and Discussion}

The objective of this analysis is to study the behavior of a steady, viscous, incompressible fluid flow through asymmetric channel of slowly varying cross-section with absorbing walls. It may be recalled that $\phi$, the phase difference, varies in the range $0 \leq \phi \leq \pi$ where $\phi=0$ corresponds to symmetric channel with waves out of phase and $\phi=\pi$ corresponds to asymmetric channel with waves are in phase. $\alpha$ represents reabsorption coefficient at the channel walls.

We discuss the effects of these parameters on the transverse velocity $(v(x, y))$, mean pressure drop $(\Delta \bar{p})$ and wall shear stress $\left(\tau_{w}\right)$ quantities. In all our numerical calculations, the following parameters are fixed as $A=-0.0628, B=0.0628, \beta_{1}=0.5, \beta_{2}=-0.5, \epsilon_{1}=0.1, \epsilon_{2}=-0.1$ and $\delta=0.1$. We take $R=1.0$ to consider low Reynolds number flow.

\subsection{The velocity $v$ :}

The transverse velocity profile of the flow is obtained by taking different values of $\phi$ at different cross-sections of the channel, for example, $x=0.1,0.5$ and 0.9. The values of $\phi$ are taken as 0 for symmetric channel, $\frac{\pi}{2}$ and $\pi$ for asymmetric channel.

Figure 2(a) displays the effect of $\phi$ on $v$ at $x=0.1$. It can be observed that as $\phi$ increases from 0 to $\pi$, the magnitude of $v$ decreases, in general. It may be remarked that the reabsorption value at the wall is fixed at $x=0.1$ and when $\phi$ increases from 0 to $\pi$, the cross sectional area reduces. This results in less $v$ values.

The cross sectional area increases as $\phi$ increases, at $x=0.5$, and this gives a mixed trend on $v$, see Figure 2(b). Note that magnitude of $v$ is less at $x=0.9$, in general, due to the reabsorption all along the wall from $x=0$ to $x=0.9$. As $\phi$ increases, the velocity $v$ increases, see Figure 2(c). 


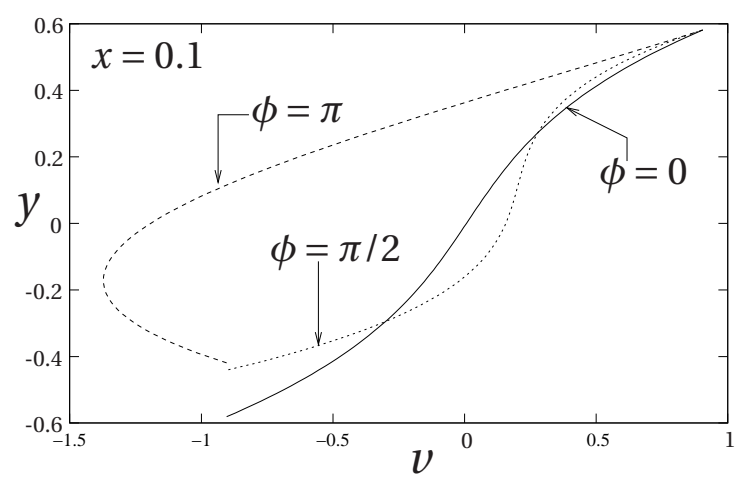

Figure 2(a): Distribution of transverse velocity $v$ with $y$ at $x=0.1, \alpha=1.0$.

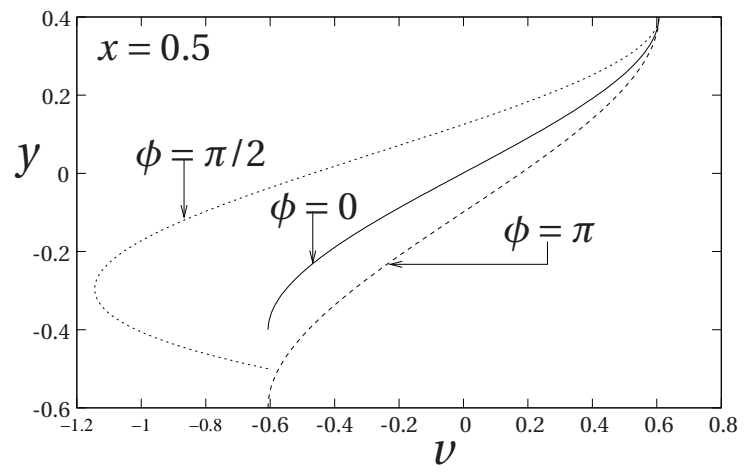

Figure 2(b): Distribution of transverse velocity $v$ with $y$ at $x=0.5, \alpha=1.0$.

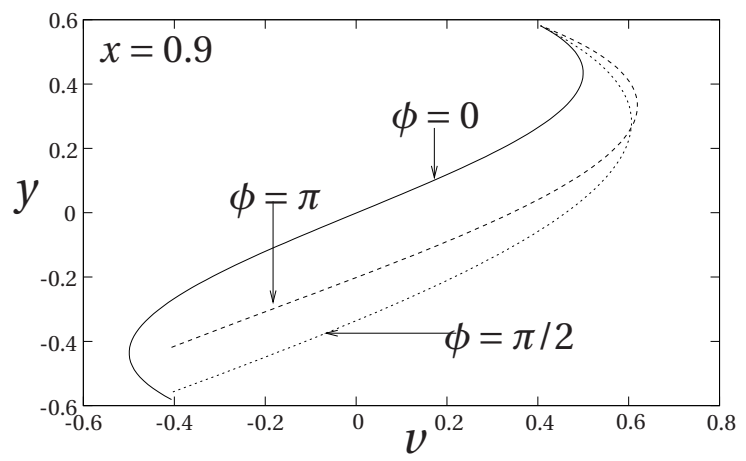

Figure 2(c): Distribution of transverse velocity $v$ with $y$ at $x=0.9, \alpha=1.0$.

These results are interpreted with earlier studies on symmetry channels and are in good agreement, especially at $\phi=0$. Moreover, we presented the effect of asymmetry on $v$ by varying $\phi$.

Generally, it can be observed from Figures 2(a) - 2(c), that as the width of the channel contracts due to the non-symmetric nature of the walls the transverse velocity decreases and as the channel width expands the velocity increases at different positions of $x$ from the entrance to the exit. Exceptionally it can be noted that at the entrance of the channel the transverse 
velocity is more for symmetric channel than the asymmetric channel, except in the middle of the channel width which is due to the wavy nature of the boundary. Furthermore, as fluid goes through the channel from entrance to the exit, the magnitude of the transverse velocity decreases.

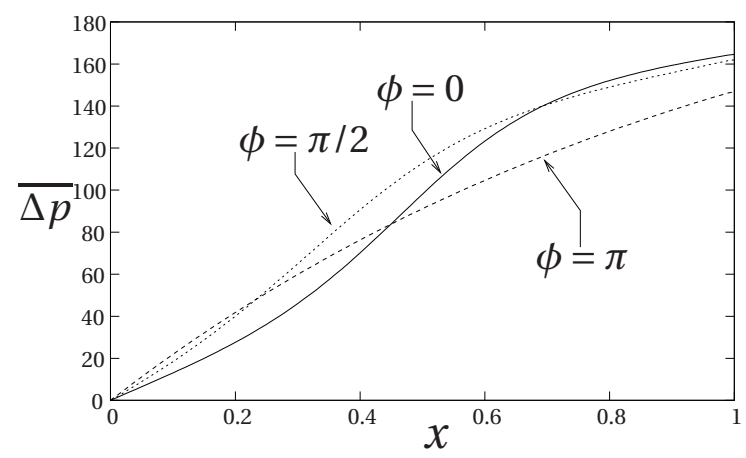

Figure 3. Distribution of mean pressure drop $\Delta p$ with $x$.

\subsection{Mean pressure drop $\Delta \bar{p}$ :}

The values of the mean pressure drop (equation (30)) over the length of the channel are calculated for different values of $\phi$ and $\alpha$. It can be noted from Figure 3 that as the width of the channel contracts the mean pressure drop increases. Particularly at the entrance of the channel the mean pressure drop for asymmetric channel is more than the symmetric channel. However, due to deep contraction in the middle of the channel the reverse is true at the end of the channel. On the other hand it is worthwhile to mention that the mean pressure drop decreases with a rise of the reabsorption coefficient whether the channel is symmetric or asymmetric or waves out of phase or waves in phase (Figures 4(a) - 4(c)).

\subsection{Magnitude of wall shear stress $\left|\tau_{w}\right|$ :}

The effects of $\phi$ and $\alpha$ on the magnitude of wall shear stress $\left(\left|\tau_{w 1}\right|\right.$ and $\left.\left|\tau_{w 2}\right|\right)$ are studied and presented graphically in Figures 5(a) - 6(c). It may be noted from Figures 5(a) and 5(b) that the magnitude of wall shear stress for both walls increase as the channel changed from symmetric to asymmetric, except in the middle of the channel where there is more contraction. This indicates that as the width of the channel decreases due to the asymmetric nature of the walls, the magnitude of the wall shear stress 1 and wall shear stress 2 increases. 


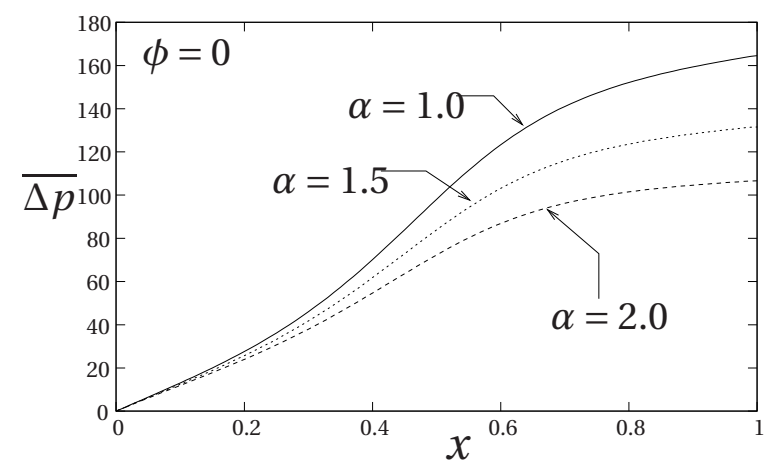

Figure 4(a): Distribution of mean pressure drop $\overline{\Delta p}$ with $x$ for $\alpha$ variation at $\phi=0$.

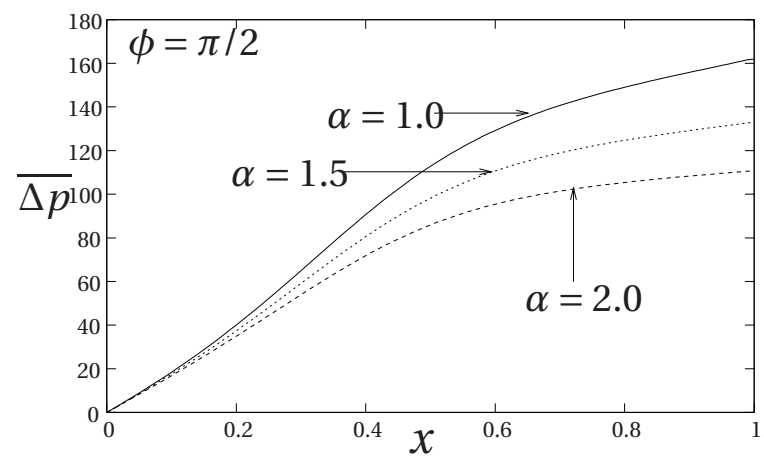

Figure 4(b): Distribution of mean pressure drop $\bar{\Delta} p$ with $x$ for $\alpha$ variation at $\phi=\frac{\pi}{2}$.

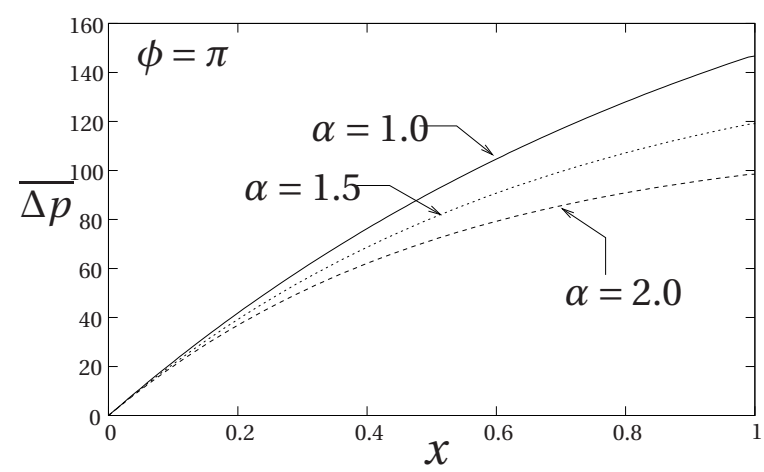

Figure 4(c): Distribution of mean pressure drop $\overline{\Delta p}$ with $x$ for $\alpha$ variation at $\phi=\pi$.

It is very important to mention that there is no such remarkable difference on the magnitude of wall shear stress 1 and wall shear stress 2 as the channel changes from symmetric to asymmetric. Even though the variation of $\phi$ doesn't have an influence in changing the boundary of the above wall, its influence is shown in the same way due to the change of the lower wall. Which might mean that the change in shape of one of the boundaries of the channel reflects in the same way to the magnitude of wall shear stress of both walls. 


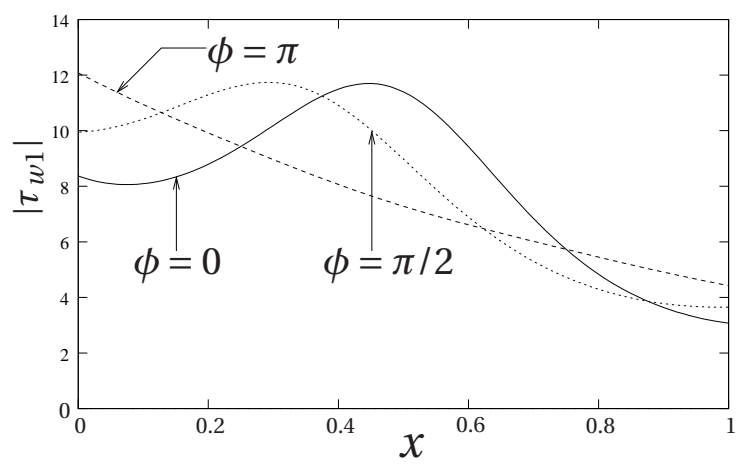

Figure 5(a): Distribution of magnitude of $\left|\tau_{w 1}\right|$ with $x$.

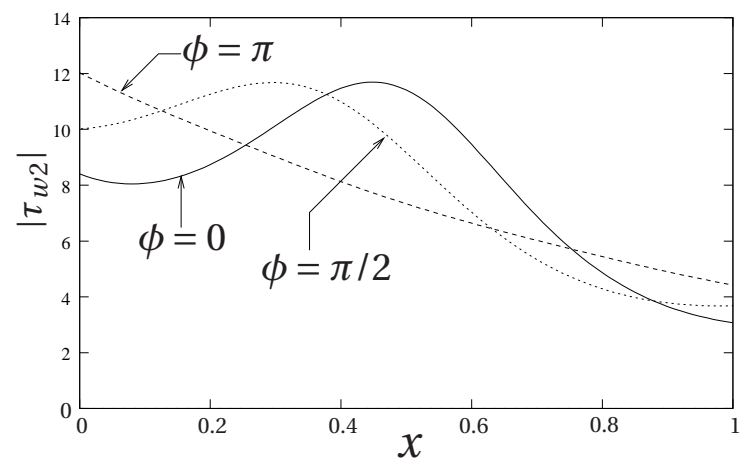

Figure 5(b): Distribution of magnitude of $\left|\tau_{w 2}\right|$ with $x$.

It can also be observed, from Figures 6(a) - 6(c), that whether the channel is symmetric or asymmetric the magnitude of wall shear stress decreases with an increase of reabsorption coefficient $\alpha$.

\section{Conclusions}

In the present study an analysis of mathematical model of steady, viscous, incompressible fluid flow in asymmetric channel of slowly varying walls has been presented with possible applications to the flow in renal tubules. The main contribution of this study is to see the effect of asymmetric nature of the channel on the flow in particular modeling of the renal tubules. It is observed that as the width of the channel expands due to the non-symmetric nature of the walls the transverse velocity increases and as the channel width contracts the velocity decreases. The mean pressure and the magnitude of the wall shear stress increases as the width of the channel contracts. Particularly at the entrance of the channel the mean pressure drop and the magnitude of wall shear stress for asymmetric channel is more than the symmetric channel. It is also worthwhile to mention that there is no such remarkable difference on the magnitude of wall shear stress 1 and wall shear stress 2 as the channel changes 
from symmetric to asymmetric. Moreover, as the reabsorption coefficient $\alpha$ rises, the magnitude of wall shear stress and the mean pressure drop decreases whether the channel is symmetric or asymmetric or waves out of phase or waves in phase.

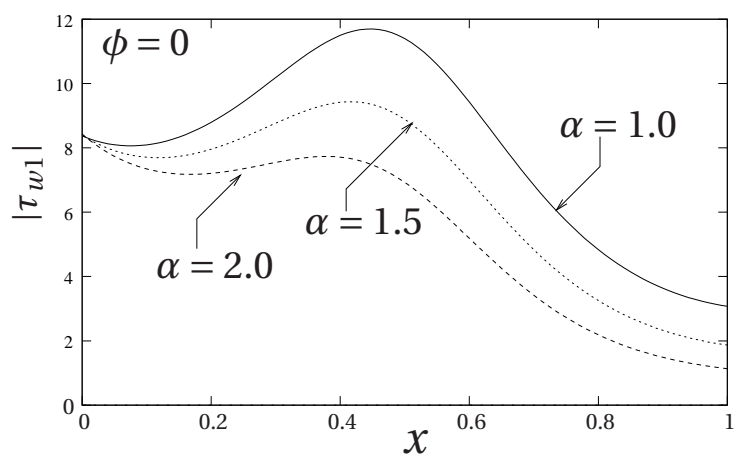

Figure 6(a): Distribution of magnitude of $\left|\tau_{w 1}\right|$ with $x$ for $\alpha$ variation at $\phi=0$.

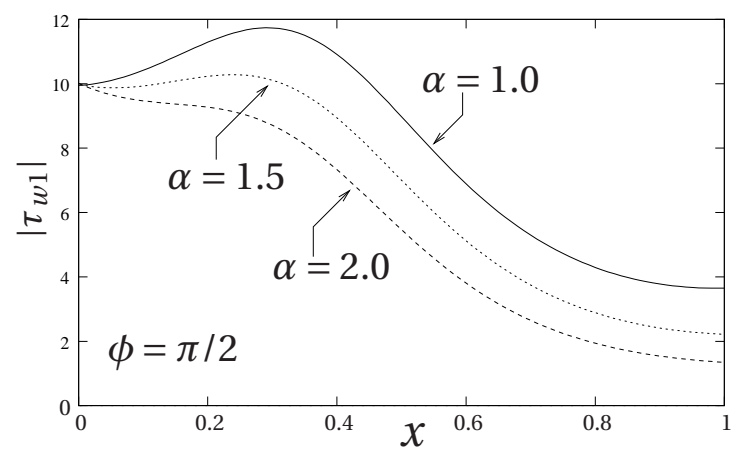

Figure 6(b): Distribution of magnitude of $\left|\tau_{w 1}\right|$ with $x$ for $\alpha$ variation at $\phi=\frac{\pi}{2}$.

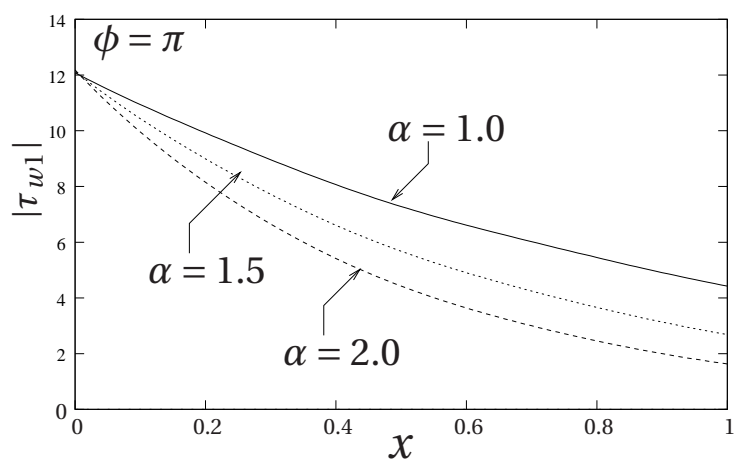

Figure 6(c): Distribution of magnitude of $\left|\tau_{w 1}\right|$ with $x$ for $\alpha$ variation at $\phi=\pi$.

\section{References}

[1] Robert I. Macey, Pressure flow patterns in a cylinder with reabsorbing walls, Bull. of Mathematical Biophysics, 25(1963), 1-9. 
[2] R. B. Kelman. A theoretical note on exponential flow in the proximal part of the mammalian nephron, Bull. of Mathematical Biophysics, 24(1962), 303-317.

[3] Robert I. Macey, Hydrodynamics in renal tubules, Bull. of Mathematical Biophysics, 27(1965), 117-124.

[4] E. A. Marshall, and E. A. Trowbridge, Flow of a Newtonian fluid through a permeable tube: The application to the proximal renal tubule, Bull. of Mathematical Biology, 36(1974), 457-476.

[5] Paul J. Palatt, Henry Sackin and Roger I. Tanner, A hydrodynamical model of a permeable tubule, J. Theor. Biol., 44(1974), 287-303.

[6] G. Radhakrisnamacharya, Peeyush Chandra and M. R. Kaimal, A hydrodynamical study of the flow in renal tubules, Bull. of Mathematical Biology, 43(1981), 151-163.

[7] Peeyush Chandra, and J. S. V. R. Krishna Prasad, Low Reynolds number flow in tubes of varying cross-section with absorbing walls, Jour. Math. Phy. Sci., 26(1992).

[8] P. Chaturani, and T. R. Ranganatha, Flow of Newtonian fluid in non-uniform tubes with variable wall permeability with application to flow in renal tubules, Acta Mechanica, 88(1991), 11-26.

[9] P. Muthu and Tesfahun Berhane, Mathematical model of flow in renal tubules. To appear in Int. Jr. App. Maths. Mech., (2010).

[10] M. Mishra and A. Ramachandra Rao, Peristaltic transport of a Newtonian fluid in an asymmetric channel, ZAMP, 54(2003), 532-550.

[11] S. Srinivas and V. Pushparaj, Nonlinear peristaltic transport in an inclined asymmetric channel, Comm. Nonlinear Sci. Numer. Simul., 13(2008), 1782-1795.

Department of Mathematics, National Institute of Technology, Warangal, Warangal - 506004. India.

E-mail: snklpm@rediffmail.com

Department of Mathematics, National Institute of Technology, Warangal, Warangal - 506004. India.

E-mail: tesfahunb2002@yahoo.com 